\title{
Functional Magnetic Resonance Imaging during Visual Perception Tasks in Adolescents Born Prematurely
}

\section{PIPARI Study Grp}

2021-03

PIPARI Study Grp , Lind , A , Haataja , L , Laasonen , M , Saunavaara , V , Railo , H , Lehtonen, T , Vorobyev , V , Uusitalo , K, Lahti , K \& Parkkola , R 2021 , ' Functional Magnetic Resonance Imaging during Visual Perception Tasks in Adolescents Born Prematurely ' , Journal of the International Neuropsychological Society , vol. 27 , no. 3 , 1355617720000867 , pp. 270-281 . https://doi.org/10.1017/S1355617720000867

http://hdl.handle.net/10138/328857

https://doi.org/10.1017/S1355617720000867

cc_by_nc_nd

acceptedVersion

Downloaded from Helda, University of Helsinki institutional repository.

This is an electronic reprint of the original article.

This reprint may differ from the original in pagination and typographic detail.

Please cite the original version. 


\section{Functional Magnetic Resonance Imaging during Visual Perception Tasks in Adolescents Born Prematurely}

Annika Lind ${ }^{\text {ab }}$, Leena Haataja ${ }^{\mathrm{c}}$, Marja Laasonen ${ }^{\text {ad }}$, Virva Saunavaara ${ }^{\mathrm{e}}$, Henry Railo ${ }^{\text {af }}$, Tuomo Lehtonen $^{\mathrm{g}}$, Victor Vorobyev ${ }^{\mathrm{h}}$, Karoliina Uusitalo ${ }^{\mathrm{i}}$, Katri Lahti ${ }^{\mathrm{i}}$, Riitta Parkkola ${ }^{\mathrm{h}}$ on behalf of the PIPARI Study Group

${ }^{\text {a }}$ Department of Psychology, University of Turku, Turku, Finland

b Turku Institute for Advanced Studies (TIAS), University of Turku, Turku, Finland

${ }^{\mathrm{c}}$ Children's Hospital, Pediatric Research Center, University of Helsinki and Helsinki University Hospital, Helsinki, Finland

${ }^{\mathrm{d}}$ Department of Speech and Language Pathology, University of Turku, Turku, Finland, Department of Otorhinolaryngology and Phoniatrics, Head and Neck Surgery, Helsinki University Hospital and University of Helsinki, Helsinki, Finland and Department of Psychology and Logopedics, University of Helsinki, Helsinki, Finland ${ }^{\mathrm{e}}$ Department of Medical Physics, Division of Medical Imaging, Turku University Hospital, Turku, Finland and Turku PET Centre, Turku University Hospital, Turku, Finland

${ }^{\mathrm{f}}$ Department of Clinical Neurophysiology, University of Turku and Turku University Hospital, Turku, Finland ${ }^{\mathrm{g}}$ Department of Ophthalmology, University of Turku and Turku University Hospital, Turku, Finland

${ }^{\mathrm{h}}$ Department of Radiology, University of Turku and Turku University Hospital, Turku, Finland

${ }^{\mathrm{i}}$ Department of Pediatric Neurology, University of Turku, Turku, Finland

Corresponding author: Annika Lind, annika.lind@utu.fi, postal address Department of Psychology, University of Turku, 20014 Turun yliopisto, Finland

Word count: Abstract 232, Manuscript text 5362 


\begin{abstract}
Objective: Impairments in visual perception are among the most common developmental difficulties related to being born prematurely, and they are often accompanied by problems in other developmental domains. Neural activation in participants born prematurely and full-term during tasks that assess several areas of visual perception has not been studied. To better understand the neural substrates of the visual perceptual impairments, we compared behavioral performance and brain activations during visual perception tasks in adolescents born very preterm (birth weight $\leq 1500$ grams or gestational age $<32$ weeks) and full-term.

Method: Tasks assessing visual closure, discrimination of a deviating figure, and discrimination of figure and ground from the Motor-Free Visual Perception Test, Third Edition were performed by participants born very preterm $(n=37)$ and full-term $(n=34)$ at 12 years of age during functional magnetic resonance imaging.
\end{abstract}

Results: Behavioral performance in the visual perception tasks did not differ between the groups. However, during the visual closure task, brain activation was significantly stronger in the group born very preterm in a number of areas including the frontal, anterior cingulate, temporal, and posterior medial parietal/cingulate cortices, as well as in parts of the cerebellum, thalamus and caudate nucleus.

Conclusions: Differing activations during the visual closure task potentially reflect a compensatory neural process related to premature birth or lesser neural efficiency, or may be a result of the use of compensatory behavioral strategies in the study group born very preterm.

Keywords: prematurity; very preterm; very low birth weight; fMRI; visual closure; visual discrimination 


\section{Introduction}

Difficulties in visual perception are among the most common neuropsychological impairments in children born prematurely (Lind, Nyman, Lehtonen, \& Haataja, 2020). Visual perception is the process of interpreting and organizing visual information. It can be divided into object processing, which involves the identification and recognition of stimuli in the environment, and spatial processing, such as the perception of location, movement and spatial relations (Adaval, Saluja, \& Jiang, 2019). Object processing has primarily been related to the ventral pathway and spatial processing to the dorsal pathway of the cortical visual system (Adaval, Saluja, \& Jiang, 2019; Kravitz, Saleem, Baker, \& Mishkin, 2011). Visual perceptual impairments can be present despite normal visual acuity (Leung, Thompson, Black, Dai, \& Alsweiler, 2018; Molloy et al., 2013), and prematurity can affect visual perception independently of cognitive level (Butcher et al., 2012; Molloy et al., 2013). Deficiencies in visual perception have, in turn, been shown to contribute to academic achievement (Johnson, Wolke, Hennessy, \& Marlow, 2011; Leung et al., 2018; Molloy et al., 2017; Perez-Roche et al., 2016). For example, in the study by Molloy et al. (2017), poorer visual perception was associated with poorer reading, spelling as well as mathematical skills in adolescents born extremely preterm or with an extremely low birth weight.

Based on a meta-analysis, the difficulties in visual perceptive abilities of participants born prematurely occur in specific areas of visual perception, particularly in the perception of line orientation (Geldof, van Wassenaer, de Kieviet, Kok, \& Oosterlaan, 2012). Global scores of tests tapping various visual perceptual subdomains have also been found to be lower in children born prematurely than in control children, but it is unclear whether the results are mediated by some of the subdomains, that is, whether performance in some of the subdomains are particularly poor and impact the global score (Geldof et al., 2012). However, deficits in visual discrimination, visual 
memory, perception of visual-spatial relationships, perception of visual form-constancy, visual sequential memory, figure-ground perception as well as in visual closure have been reported in children with a very low birth weight (Davis, Burns, Wilkerson, \& Steichen, 2005). In a study by Perez-Roche et al. (2016), several areas related to visual perception were compared at ages 5-13 between children born before gestational week 37, children born full-term, children who were small for gestational age (SGA) and children who were not SGA. Discrimination of figure and ground and visual motor integration were found to be poorer in children born prematurely than in children born full-term. Visual memory and perception of form constancy, figure- ground and visual closure were, in turn, poorer in children that were SGA. Hence, Perez-Roche et al. suggest that preterm birth affects the dorsal visual stream in particular, while being born SGA might result in more diffuse damage.

Overall, Functional Magnetic Resonance Imaging (fMRI) during completion of cognitive tasks has increased our understanding of the neurobiological substrates associated with developmental difficulties in children born prematurely. Brain activation in children and adults born prematurely has shown to differ from that of control children born full-term during tasks assessing, for example, executive functioning (de Kieviet et al., 2014), attention and working memory (Daamen et al., 2015), visual memory (Brittain et al., 2014; Chaminade, Leutcher, Millet, \& Deruelle, 2013), verbal memory (Salvan et al., 2013), and language functions (Ettinger- Veenstra et al., 2017). In some of the earlier studies on brain activation during various cognitive tasks, different brain regions have been activated in participants born prematurely and full-term (Chaminade et al., 2013), while other studies show that the same regions are activated in participants born prematurely and in controls, but that activation in these regions is weaker or stronger in those born prematurely (Daamen et al., 2015; Ettinger-Veenstra et al., 2017). Although visual perceptual difficulties are common in individuals born prematurely, there are, to our knowledge, no studies comparing fMRI activations 
in participants born prematurely and full-term during tasks that assess several areas of visual perception. Dorn et al. (2014) studied visuospatial processing using fMRI and a pattern matching task in children and adolescents who had been born before gestational week 33, but the focus in their study was on the consequences of early postnatal human cytomegalovirus infection and the ages of the participants varied from 7 to 18 years. They found that neural activation in the occipital cortex differed between the prematurely born and control group during the visuospatial task.

It is unclear how difficulties in specific areas of visual perception relate to brain regions associated with visual and neuropsychological functions. Thus, there is a need for detailed studies on different areas of visual perception and their underlying mechanisms. The aim of the present study was to assess if fMRI activations differ between adolescents born very preterm and full-term during tasks assessing visual closure, discrimination of a deviating figure, and discrimination of figure and ground. Our hypothesis was that the adolescents who had been born very preterm would show poorer performance in visual perception tasks compared to the group born full-term, and that the neural activation pattern would differ between the groups, possibly due to suboptimal development of neural networks in children born very preterm. In some fMRI studies, only specific brain regions of interest selected in advance were analyzed (Gimenez et al., 2005; Griffiths et al., 2014). We chose to not limit the areas analyzed, since no previous studies with a comparable study protocol were found, and since relatively diffuse neural networks have been activated in participants born prematurely in earlier fMRI studies on cognitive processes. 


\section{Materials and methods}

\section{Participants}

This study is part of the multidisciplinary PIPARI project (Development and Functioning of Very Low Birth Weight Infants from Infancy to School Age). All infants born with a very low birth weight $(\leq 1500 \mathrm{~g})$ at Turku University Hospital in Finland between 2001 and 2006 who lived in the hospital catchment area and whose parents spoke and understood written Finnish or Swedish were eligible. From the beginning of 2004, the inclusion criteria were expanded to include all infants born $<32$ weeks of gestation, regardless of their birthweight. Infants with major congenital anomalies or syndromes or chromosomal anomalies were excluded. From this sub-study also participants with known significant cognitive impairment (full scale intelligence quotient $<70$ ), cerebral palsy, severe hearing impairment (loss of hearing requiring amplification in at least one ear), and/or visual impairment (binocular visual acuity $<1.0$ on the Snellen scale, high astigmatism of over 1 diopter, amblyopic eyes or crowding phenomenon) were also excluded. No participants with major brain pathology at 12 years of age were included (please see Brain imaging section for definition of major brain pathology). This sub-study includes 37 adolescents born very preterm in 2004-2006 who participated in fMRI at 12 years of age (Figure 1). Three of the participants included had no data available from ophthalmological examination. A control group of 34 adolescents born full-term and participating in the PIPARI project, born 2003-2004 and recruited as described in Lind et al., (2011), was also included. The participants included as controls had no major findings in structural magnetic resonance imaging (MRI) at 12 years of age (please see Brain imaging section for definition of major brain pathology). The characteristics of the participants born very preterm $(n=37)$ and the control group $(n=34)$ are presented in Table 1 . The study has been approved by the Ethical Committee of the Hospital District of Southwest Finland. Informed consent was obtained from the parents, and at 12 years of age, the participants also gave their own written informed consent after receiving written information. 


\section{[INSERT FIGURE 1 AND TABLE 1 HERE]}

\section{Visual perception task}

Visual perception at 12 years of age was assessed with items of the Motor-Free Visual Perception Test, Third Edition (MVPT-3; Colarusso \& Hammill, 2003). An electronic version of the test suitable for fMRI, was created with permission from the publisher, Academic Therapy Publications. The items were presented on a screen during fMRI, each item had four possible answers and the participants answered by pressing one of four response buttons. A button press ended stimulus presentation and started gaze fixation screen. Therefore, natural variability of response time would introduce a jitter in stimulus-onset asynchrony. The items were presented in three series. The first series assessed visual closure, the ability to perceive a whole figure when only fragments of it are presented, and it included MVPT-3 items 22-34 and 56-60. The second series assessed discrimination of a deviating figure and included items 35-45. The third series assessed discrimination of figure and ground and included items 51-55. The stimuli were black-and-white line drawings and designs. If not interrupted by a response, each item was presented for 9 seconds (visual closure and discrimination of a deviating figure) or 15 seconds (discrimination of figure and ground). Each session started with a 17-second gaze-fixation baseline period, then the task and gaze-fixation periods alternated (Figure 2). The relatively long gaze-fixation condition ensured considerable fading of task-related blood-oxygenation-level dependent (BOLD) signal before the next stimulus. Additionally, it provided enough scan data for the control condition even in cases of late response times exceeding the stimulus presentation period. Before fMRI, 1-2 training items per series were administered, and the instructions were orally repeated before each series during the imaging session. The training items were not included in the actual task. In addition to brain region activation, reaction time and the accuracy of responses for each series were registered. In trials where participants occasionally gave multiple button presses, the earliest response was used to 
define accuracy and response time. If the participant did not answer during stimulus presentation, earliest response during the following gaze fixation period was counted. The MVPT-3 was chosen to present study as the tasks were found appropriate for this age group, as the tasks did not require motor skills, as the test allows assessment of various specific sub-domains of visual perception, as a modification to an electronic version was feasible, and as the test is widely used and accessible and hence allows replication of our study.

\section{[INSERT FIGURE 2 HERE]}

\section{Brain imaging}

A brain MRI at term equivalent age was performed with a 1.5T Philips Intera (Philips Medical Systems, Best, The Netherlands) for infants born very preterm. The findings were categorized into three groups to describe the level of brain pathology (Table 1): 1) Normal findings consisting of normal brain signal intensity and anatomy of the cortex and cortical gyration pattern, basal ganglia and thalami, posterior limb of internal capsule, white matter, germinal matrix, corpus callosum, cerebellum, pons and medulla oblongata, a width of extracerebral space of less than $5 \mathrm{~mm}$ and a ventricular/brain ratio of less than $0.35 ; 2$ ) Minor pathologies consisting of the consequences of intraventricular hemorrhages of grades 1 and 2, such as minor linear T2 hyperintensities of the caudothalamic grooves or caudothalamic groove cysts smaller than $3 \mathrm{~mm}$, an extracerebral space width of $5 \mathrm{~mm}$, and a ventricular/brain ratio of 0.35 ; 3) Major pathologies consisting of the consequences of intraventricular hemorrhages of grades 3 and 4, cystic or cystic and hemorrhagic white matter lesions, as well as cystic or cystic and hemorrhagic lesions of the cortex, basal ganglia, thalamus, internal capsule, corpus callosum or cerebellum, focal T1 hyperintensities of deep or 
periventricular white matter corresponding to gliosis and extracerebral space witdht increased by $>5$ $\mathrm{mm}$, a ventricular/brain ratio of $>0.35$, ventriculitis, or focal infarctions.

The MRI study of participants born very preterm or full-term at 12 years of age included both structural data collection and functional imaging of brain activation during visual perception tasks using 3T Philips Ingenuity TF PET/MR (Philips, Amsterdam, Netherlands). Three imaging protocols were used for clinical assessment: a T2-weighted set of axial slices with a $4.82 \mathrm{~s}$ Repetition Time (TR) and an $80 \mathrm{~ms}$ Echo Time (TE) and $3 \mathrm{~mm}$ slice thickness; Coronal Fluid Attenuation Inversion Recovery sagittal imaging with 10 s TR, 2.8 s Inversion Time, TE of 125 ms, a slice thickness of $4 \mathrm{~mm}$; and a 3D T1 set of sagittal slices with $8.1 \mathrm{~ms}$ TR, $3.7 \mathrm{~ms}$ TE and isotropic $1 \mathrm{~mm}$ voxel. The last mentioned was also used in fMRI data processing. The MRI findings at the age of 12 were also categorized into three groups (Table 1): 1) Normal findings consisted of normal brain signal intensity and anatomy of the cortex and cortical gyration pattern, basal ganglia and thalami, internal and external capsule, white matter, corpus callosum, cerebellum, pons and medulla oblongata and normal cerebrospinal fluid spaces; 2) Minor pathologies such as mild prominence of one of the four lateral ventricular horns without brain parenchyma pathologies or minor punctuate cerebral white matter T1 hyperintensity; 3) T2 hyperintensity of the cerebral or cerebellar parenchyma corresponding to focal hemosiderin collection, symmetric or asymmetric white matter damage corresponding to white matter gliosis, marked dilatation of the ventricles or marked dilatation of the cortical cerebrospinal fluid spaces or signs of infarcts or cystic and/or hemorrhagic white or grey matter damage, were considered as major pathologies. All magnetic resonance images were assessed by an experienced neuroradiologist.

Functional MRI data sensitive to a BOLD signal was collected for the whole brain volume (35 axial slices of $4 \mathrm{~mm}$ thickness) using a single-shot sequence for $\mathrm{T} 2 *$-weighted echo planar imaging with 
a $2.0 \mathrm{~s}$ TR, $20 \mathrm{~ms} \mathrm{TE}, 75^{\circ}$ flip angle, $80 \times 80$ matrix, and $3 \mathrm{~mm}$ in-plane resolution. Each fMRI session included four dummy scans to allow for T1 equilibration effects. Each participant completed three fMRI runs corresponding to each task (191-231 scans, 124-147 scans, 66-90 scans for visual closure, discrimination of a deviating figure, and discrimination of figure and ground series, respectively). The presentation software (Neurobehavioral Systems, Inc., Albany CA, USA) was used to synchronize BOLD data acquisition with stimulus delivery as well as for response data collection.

In the fMRI study, spherical refractive errors were compensated for with adjustable MRIcompatible binoculars. Based on an ophthalmological examination of the participants born very preterm, performed at 11 years of age, visual acuity was normal with stable fixation in all the examined participants that met the inclusion criteria.

\section{Data analyses}

Background data and task performance data were analyzed with the SPSS 24.0 software (IBM, Armonk, NY, USA). For comparisons between the group born very preterm and the control group, the chi-square test, the Fisher's exact test, the t-test for independent samples or the Mann-Whitney U-test was used, as appropriate. Effect sizes were calculated as Cohens's d when the t-test for independent samples was used and as eta squared when the Mann-Whitney U-test was used. Age at assessment and response time for discrimination of a deviating figure were analyzed with the t-test and the other continuous variables were analyzed with the Mann-Whitney U-test. P-values $<0.05$ were considered statistically significant.

The MRI data were processed with the Statistical Parametric Mapping (SPM12, Wellcome 
Department of Cognitive Neurology, London, UK) and the toolboxes implemented in Matlab (MathWorks Inc., Natik MA, USA). The within-participant pre-statistical fMRI data processing included head motion correction with a two-step realignment procedure by registering each volume to the realigned mean image. Next, images were corrected for slice timing using the middle slice for reference and co-registered with the high-resolution T1-weighed image for each participant. In order to spatially normalize the functional data while taking into account potential systematic structural differences between early adolescent and adult brains, a customized sample-specific structural brain template was created with the Computational Anatomy Toolbox CAT12 software (Gaser \& Dahnke, 2016). Each T1-weighted structural volume was initially segmented into grey matter (GM), white matter, and cerebrospinal fluid using age-specific tissue probability maps created with the Template-O-Matic TOM8 software (Wilke, Holland, Altaye, \& Gaser, 2008). The resulting tissue class images, affinely registered to the Montreal Neurological Institute MNI152 standard brain space, were used to create the customized template by applying the Diffeomorphic Anatomic Registration Through Exponentiated Lie algebra algorithm (DARTEL; Ashburner, 2007). The customized sample-based template was built using segmented structural data of 58 participants with equal contribution from both groups. Then the deformation fields representing spatial transformations of each individual GM image to the space of the customized template were calculated. The deformation fields were applied to functional images to warp them into the space of the customized template. The resulting images were also resliced to a $3 \mathrm{~mm}$ cubic voxel and smoothed with an $8 \mathrm{~mm}$ Gaussian kernel.

One control participant and one participant born prematurely were excluded from fMRI analysis as having either wide cisterna magna, which falls within normal variation, or a large susceptibility artifact in the ventral frontal area, respectively. Functional MRI data from several participants were excluded from statistical analysis due to excessive head motion as follows: three control and three 
participants born very preterm for the visual closure series; four control and six very preterm for the discrimination of a deviating figure series; and two control and three very preterm for the discrimination of figure and ground series. Maximal between-scan head motion in these cases exceeded at least one of the following criteria: $3 \mathrm{~mm}$ in translation or 3 degrees in rotation. In addition, one control participant was excluded because of multiple extra response- related activity also extending to the gaze-fixation period in discrimination of a deviating figure and of figure and ground, and one participant born very preterm was rejected only for the visual closure task analysis due to a stimulus presentation problem during the task. In total, in the control group, all three tasks were excluded from three participants, two tasks from two participants and one task from one participant. In the group born very preterm, all three tasks were excluded from three, two tasks from two and one task from three participants. Accordingly, fMRI data from 28 control participants and 29 participants born very preterm were used. The resulting groups for fMRI analyses included 30/32, 28/30, and 30/33 (control/prematurely born) participants for the three visual perception tasks, respectively. Proportions of female participants in the tasks were 16/13, 15/13, and 17/13 (control/prematurely born). For each of the tasks, the first-level General Linear Model implemented in the SPM included a task condition of variable-length epochs equal to response times (Grinband, Wager, Lindquist, Ferrera, \& Hirsch, 2008), a separate condition for trials with a missing response with duration equal to default stimulus presentation time, and an implicitly modelled gaze fixation condition of $17 \mathrm{~s}$ duration, allowing for almost complete fading of hemodynamic response from preceding task-related activity. In order to minimize the impact of head motion, the model also included dummy regressors for scans with excessive movements, six realignment parameters, and a regressor for the Euclidean norm of the parameters as provided by the Artifact Detection Tools (ART, Susan Whitfield- Gabrieli, MIT, US) software with the following criteria for outlier scans: scan-to-scan changes in global mean $\mathrm{z}>4$; translation $>2 \mathrm{~mm}$; rotation $>1$ degree $(0.0175 \mathrm{rad})$. A canonical hemodynamic response function was used to convolve stimulus functions and a high-pass 
filter with a cut-off period of 128 seconds was applied to account for low frequency scanner drift. Contrast images containing activation differences between the visual task and gaze fixation were calculated for each participant and fed into the second-level group analysis, treating participants as a random factor. The analysis was confined both by implicit masking and by a GM mask, the latter was created by $8 \mathrm{~mm}$ Gaussian smoothing of the GM template followed by intensity thresholding with a value of 0.3 . First, exploratory analysis of activation patterns for each visual task was performed within each group with one-sample t-tests and a voxel threshold of $p<0.05$ after familywise error (FWE) correction for multiple comparisons. Similarity in group-specific brain activation across tasks was obtained through the extraction of overlap between binarized maps of significant activation. For the estimation of the group difference in each visual task, the Threshold-Free Cluster Enhancement (TFCE; Smith \& Nichols, 2009) ), a permutation-based non-parametric method, as implemented in the TFCE Toolbox (Christian Gaser, Jena University Hospital, Germany), was applied to estimate a combined voxel-cluster significance of the between-group differences using the default values for voxel and cluster weight $(\mathrm{H}=2.0, \mathrm{E}=0.5), 5000$ permutations and an FWE corrected $p<0.05$ significance threshold. In addition, a few clusters containing less than 5 voxels with $\mathrm{p}$ values in the range of $0.048-0.049$ were not taken into consideration. Statistical analysis that would include all three task series into a single model was not performed due to differences in stimulus timing, task difficulty levels / response times, and amount of data collected between tasks leading to an unbalanced design. 


\section{Results}

The background characteristics of the group consisting of adolescents born very preterm and the control group are shown in Table I. The number of girls/boys, $\left[\chi^{2}(1)=1.633, p=.201\right]$, maternal education $\left[\chi^{2}(1)=0.485, p=.486\right]$, paternal education $\left[\chi^{2}(1)=2.334, p=.127\right]$, age at assessment, $[t(60.354)=0.598, p=.552, d=.300]$, or handedness (Fisher's exact test, $p=.615)$, did not differ significantly between the participants born very preterm and the control group. Results in the visual perception task are presented in Table 2. Neither the accuracy nor response time of correct responses differed significantly between the group born very preterm and the control group in any of the task series. Exclusion of one control child who did not give any correct responses in visual closure or discrimination of a deviating figure, did not change the results to significant.

\section{[INSERT TABLE 2 HERE]}

Exploratory analysis of activation difference between the task and gaze fixation conditions within each task and group revealed a fairly similar activation pattern across the tasks and groups (Figure 3). The areas of overlap across all three tasks included bilateral medial and lateral parts of the occipital cortex, anterior cingulate, superior parietal, anterior insular and lateral premotor and prefrontal cortices, and the cerebellum. There was also activation in the caudate head and thalamus, bilateral for the control group and right-sided for the group born very preterm. The common deactivation pattern for both groups included areas of what is considered as the default mode network: the bilateral posterior cingulate, lateral inferior parietal and temporal areas. Deactivation in the ventromedial prefrontal cortex was present in the control group only.

\section{[INSERT FIGURE 3 HERE]}


Regarding fMRI, between-group comparisons of activation during the visual closure task revealed stronger activation in participants born very preterm than in the control group in the thalamus and caudate bilaterally, in the left dorsolateral prefrontal, orbitofrontal and premotor cortices, in the polar and middle part of the temporal lobe, and in the cerebellum (Table 3, Figure 4). The midline areas were located in the premotor, prefrontal and cingulate cortices, as well as at the border of the posterior cingulate and precuneus. Though all the midline clusters had their peaks in the left hemisphere, two clusters (pre-supplementary motor area and anterior cingulate) appeared to also extend to the right hemisphere (Figure 4, slices with $\mathrm{z}$ coordinates 30 and 48). The opposite group comparison did not yield any significant difference. The fMRI activations during the discrimination of a deviating figure and discrimination of figure and ground tasks did not differ significantly between the groups.

[INSERT TABLE 3 AND FIGURE 4 HERE] 


\section{Discussion}

In this study, performance in tasks assessing visual closure, discrimination of a deviating figure, discrimination of figure and ground and brain activation during the tasks were compared between adolescents born very preterm and full-term. Task performance was comparable in all three tasks, and all tasks evoked a highly overlapping pattern of brain activity in both groups. Nevertheless, brain activation during the visual closure task differed significantly between the participants born very preterm and the control group. Activation was stronger in the participants born very preterm during the visual closure task in several areas, including frontal, anterior cingulate, temporal, and posterior medial parietal/cingulate cortices as well as in parts of the cerebellum, thalamus and caudate nucleus. We hypothesized that both visual perception task performance and brain activation during those tasks would differ between the participants born very preterm and the control group, and it was unexpected that task performance in the assessed visual perception domains did not diverge significantly.

In order to explicitly validate the tasks at the level of neural activation, we performed extraction of within group task-specific activation patterns by comparing task and baseline conditions. It has been shown that a passive control condition may be related to uncontrolled mental activity, for example concerning semantic areas (Binder, Swanson, Hammeke, \& Sabsevitz, 2008). We did, however, not expect considerable similarity between activity of interest in the control and task condition, given that our tasks were related to visual analysis. Indeed, all "task vs. gaze-fixation" contrasts demonstrated rather expected patterns of activation for such tasks. Moreover, in spite of differences between the tasks, the patterns demonstrated a considerable similarity. 
Dorn et al. (2014) also found neural activation differences during visuospatial pattern matching task in participants born before gestational week 33 and control participants, but in their study, activation differed in the occipital cortex only. In our study, no activation differences were seen in the occipital cortex, which may be related to the strict exclusion of participants with visual impairments. In an fMRI study by Murner-Lavanchy et al. (2014a), children born before gestational week 32 and/or with a birth weight below $1500 \mathrm{~g}$ and the control group had equal visuospatial working memory performance, but the children born prematurely showed less involvement of the right middle frontal gyrus and higher activation in the superior frontal regions than controls. Also in our study activation was increased in frontal areas in the group born very preterm, while task performance was similar to the control group.

Differences in neural activation between participants born prematurely and control participants have been explained by compensatory processes following aberrant brain development or dysfunction related to prematurity (Brittain et al., 2014; Ettinger-Veenstra et al., 2017). Ettinger-Veenstra et al. studied language functions in adolescents born with a very low birth weight during fMRI and found increased activation in some regions and decreased activation in others compared to controls born full-term. Despite altered neural activation during the language tasks, accuracy and reaction times in the tasks did not differ between participants born prematurely and full-term. Thus, the authors suggest compensatory neural mechanisms through recruitment of additional brain regions regarding language functions in adolescents born prematurely, a strategy that appeared to be effective since task performance did not differ. This is in line with our results, since neural activation during the visual closure task was significantly stronger in several regions in participants born very preterm, while task performance was comparable between the participants born very preterm and the control group. 
Differences in brain activation in children born prematurely and full-term have also been explained by delayed development of neural networks in children born prematurely (Murner-Lavanchy et al., 2014a, 2014b). In the study by Murner-Lavanchy et al. (2014b) including 7-12-year old children born before gestational week 32 and/or with a birth weight below 1500g, younger children showed a bilateral and atypical language network while the older ones showed left-sided language organization. There were no age-related changes in controls. Comparably, in the work by MurnerLavanchy et al. (2014a), brain activation network during a visuospatial working memory task of 7 12- year old children born prematurely was similar to the controls in the older and high-performing participants. The younger and low-performing participants had, in turn, an atypical working memory network, and higher intensity of activation in the frontal areas, which was interpreted as less neural efficiency since the task performance did not differ between the groups. As the more typical network appeared to be established with increasing age, it can as well be considered as a compensational process. However, other studies have shown differences in brain activation between prematurely born and control groups also in adulthood during tasks assessing learning and memory of visual material (Brittain et al., 2014; Froudist-Walsh et al., 2015; Narberhaus et al., 2009). Instead of reflecting compensatory neural processes, the activation difference between the adolescents born prematurely and full-term in our study might likewise be the result of behavioral compensation, such as the use of different behavioral strategies during task performance. Compensatory neural mechanisms were indeed questioned in a study by Morcom and Henson (2018). Increased task-related prefrontal activation is often observed in healthy aging adults and is viewed as a compensatory process aimed at maintaining cognitive function, but was by Morcom and Henson suggested to reflect less specific or less efficient activation caused by structural and neurochemical changes rather than a compensatory mechanism. 
Previous studies have revealed more specific, rather than global, deficits in visual perceptive abilities of children born prematurely (Geldof et al., 2012). In our study, different aspects of visual perception were separately analyzed, but performance in each task was equal to that of the control group. It is, however, interesting that there were notable differences in accuracy rates between the tasks. As for the visual closure task, accuracy was $71 \%$, while the amount of corrects responses in the discrimination of figure and ground task was $29 \%$ in the group born very preterm. The percentages of correct responses were comparable in the control group, and the accuracy differences are hence likely to reflect the complexity or difficulty level of the tasks. The lack of differences in task performance at 12 years of age in our study can be a result of developmental catch-up in the group born very preterm and the performance in our sample may have diverged at an earlier age. In present study, the group born very preterm did not include participants with neurodevelopmental or visual impairments or with major brain pathology, and it is possible that the task performance between the groups would have differed more if less strict inclusion criteria had been applied. However, inclusion of participants with known deviations in brain development would have biased the interpretation of the fMRI findings. It is also possible that visual perceptual deficits in children and adolescents born prematurely are more pronounced in other sub-domains of visual perception than those assessed in our study.

The main limitations of the present study are related to the moderate number of items per task. It is possible that series with more items would have differentiated the task performance between the groups. The number of items also impacts the fMRI analyses, and the fact that activation differed during one of three tasks might be a reflection of task length. In our study, activation differed broadly during the visual closure task consisting of 18 items, but not during the tasks including 11 and five items. A small number of trials per task can increase sensitivity to noise factors providing a higher within-group signal variance, thus precluding reliable detection of group difference. The 
division of the MVPT-3 items into three series in our study might overall be questioned; the series that aimed to assess visual discrimination included both discrimination of different shapes and discrimination of rotations. In addition, there were some minor differences in the amount of usable fMRI data between the participants born very preterm and full-term. However, as there is a lack of studies examining the neural correlates of potential deficits in visual perception in participants born prematurely, our results are valuable. The group born prematurely was well defined in our study, without neurodevelopmental and visual impairments or major structural brain deviations at the time of assessment, all participants were assessed when they were of the same age, and refractive errors were compensated for in the fMRI.

In conclusion, in this study, behavioral performance in visual perception tasks was comparable in adolescents born very preterm and full-term. In turn, brain activation differed during the visual closure task even though the task performance did not. Activation in a number of brain areas was stronger in the group born very preterm. The causes for differing activations cannot be established based on our study, but stronger activations potentially reflect a compensatory neural process due to prematurity, resulting in task performance equal to that of the group born full-term, or lesser neural efficiency in participants born very preterm, or else, compensatory behavioral task performance strategies. 


\section{Acknowledgements}

The PIPARI Study Group includes, Mikael Ekblad, Satu Ekblad, Eeva Ekholm, Linda Grönroos, Leena Haataja, Mira Huhtala, Jere Jaakkola, Max Karukivi, Pentti Kero, Riikka Korja, Katri Lahti, Helena Lapinleimu, Liisa Lehtonen, Tuomo Lehtonen, Marika Leppänen, Annika Lind, Mari Koivisto, Mira Mattsson, Jonna Maunu, Petriina Munck, Laura Määttänen, Pekka Niemi, Anna Nyman, Riitta Parkkola, Liisi Ripatti, Päivi Rautava, Katriina Saarinen, Tiina Saarinen, Susanna Salomäki, Virva Saunavaara, Sirkku Setänen, Matti Sillanpää, Suvi Stolt, Päivi Tuomikoski, Karoliina Uusitalo and Milla Ylijoki.

Declarations of interest: none

Funding: This work was supported by governmental funding for research in university hospitals ('ERVA' funding); Foundation for Paediatric Research; and Turku Institute for Advanced Studies 


\section{References}

Adaval, R, Saluja, G, Jiang, Y (2019). Seeing and thinking in pictures: A review of visual information processing. Consumer psychology review, 2, 50-69.

Ashburner, J. (2007). A fast diffeomorphic image registration algorithm. Neuroimage, 38, 95-113.

Binder JR, Swanson SJ, Hammeke TA, Sabsevitz DS (2008). A comparison of five fMRI protocols for mapping speech comprehension systems. Epilepsia, 49,1980-1997.

Brittain, P. J., Froudist-Walsh, S., Nam, K. W., Giampietro, V., Karolis, V., Murray, R. M.,...Nosarti, C. (2014). Neural compensation in adulthood following very preterm birth demonstrated during a visual paired associates learning task. Neuroimage Clinical, 6, 54-63.

Butcher, P. R., Bouma, A., Stremmelaar, E. F., Bos, A. F., Smithson, M., Van Braeckel, K. N. (2012). Visuospatial perception in children born preterm with no major neurological disorders. Neuropsychology, 26, 723-734.

Chaminade, T., Leutcher, R. H., Millet, V., Deruelle, C. (2013). fMRI evidence for dorsal stream processing abnormality in adults born preterm. Brain and Cognition, 81, 67-72. 
Colarusso, R., Hammill, D., (2003). Motor-free Visual Perception Test, Third Edition. Academic Therapy Publications, Novata, CA.

Daamen, M., Bauml, J. G., Scheef, L., Sorg, C., Busch, B., Baumann, N.,...Boecker, H. (2015). Working memory in preterm-born adults: Load-dependent compensatory activity of the posterior default mode network. Human Brain Mapping, 36, 1121-1137.

Davis, D. W., Burns, B. M., Wilkerson, S. A., Steichen, J. J. (2005). Visual perceptual skills in children born with very low birth weights. Journal of Pediatric Health Care, 19, 363-368.

Dorn, M., Lidzba, K., Bevot, A., Goelz, R., Hauser, T. K., Wilke, M. (2014). Long-term neurobiological consequences of early postnatal hCMV-infection in former preterms: A functional MRI study. Human Brain Mapping, 35, 2594-2606.

van Ettinger-Veenstra, H., Widen, C., Engstrom, M., Karlsson, T., Leijon, I., Nelson, N. (2017). Neuroimaging of decoding and language comprehension in young very low birth weight (VLBW) adolescents: Indications for compensatory mechanisms. PloS ONE, 12, e0185571.

Froudist-Walsh, S., Karolis, V., Caldinelli, C., Brittain, P. J., Kroll, J., Rodriguez-Toscano, E.,...Nosarti, C. (2015). Very early brain damage leads to remodeling of the working memory system in adulthood: A combined fMRI/Tractography study. The Journal of Neuroscience, 35, 15787-15799. 
Gaser, G., Dahnke, R. (2016). CAT - A Computational Anatomy Toolbox for the Analysis of Structural MRI Data. HBM 2016, 336-348.

Geldof, C. J., van Wassenaer, A. G., de Kieviet, J. F., Kok, J. H., Oosterlaan, J. (2012). Visual perception and visual-motor integration in very preterm and/or very low birth weight children: A meta-analysis. Research in Developmental Disabilities, 33, 726-736.

Gimenez, M., Junque, C., Vendrell, P., Caldu, X., Narberhaus, A., Bargallo, N.,...Mercader, J.M. (2005). Hippocampal functional magnetic resonance imaging during a face-name learning task in adolescents with antecedents of prematurity. Neuroimage, 25, 561-569.

Griffiths, S. T., Aukland, S. M., Markestad, T., Eide, G. E., Elgen, I., Craven, A. R., Hugdahl, K. (2014). Association between brain activation (fMRI), cognition and school performance in extremely preterm and term born children. Scandinavian Journal of Psychology, 55, 427-432.

Grinband, J., Wager, T.D., Lindquist, M., Ferrera, V.P., Hirsch, J. (2008). Detection of time-varying signals in event-related fMRI designs. Neuroimage, 43, 509-520. 
Johnson, S., Wolke, D., Hennessy, E., Marlow, N. (2011). Educational outcomes in extremely preterm children: Neuropsychological correlates and predictors of attainment. Developmental Neuropsychology, 36, 74-95.

de Kieviet, J. F., Heslenfeld, D. J., Pouwels, P. J., Lafeber, H. N., Vermeulen, R. J., van Elburg, R. M., Oosterlaan, J. (2014). A crucial role for white matter alterations in interference control problems of very preterm children. Pediatric Research, 75, 731-737.

Kravitz, D. J., Saleem, K. S., Baker, C. I., \& Mishkin, M. (2011). A new neural framework for visuospatial processing. Nature reviews. Neuroscience, 12, 217-230.

Leung, M. P., Thompson, B., Black, J., Dai, S., Alsweiler, J. M. (2018). The effects of preterm birth on visual development. Clinical \& Experimental Optometry, 101, 4-12.

Lind, A., Korkman, M., Lehtonen, L., Lapinleimu, H., Parkkola, R., Matomaki, J.,...the PIPARI Study Group (2011). Cognitive and neuropsychological outcomes at 5 years of age in preterm children born in the 2000s. Developmental Medicine and Child Neurology, 53, 256-262.

Lind, A., Nyman, A., Lehtonen, L., \& Haataja, L. (2020). Predictive value of psychological assessment at five years of age in the long-term follow-up of very preterm children. Child Neuropsychology, 26, 312-323. 
Molloy, C. S., Wilson-Ching, M., Anderson, V. A., Roberts, G., Anderson, P. J., Doyle, L. W. (2013). Visual processing in adolescents born extremely low birth weight and/or extremely preterm. Pediatrics, 132, e704-12.

Molloy, C. S., Di Battista, A. M., Anderson, V. A., Burnett, A., Lee, K. J., Roberts, G.,...Doyle, L.W. (2017). The contribution of visual processing to academic achievement in adolescents born extremely preterm or extremely low birth weight. Child Neuropsychology, 23, 361-379.

Morcom, A.M., Henson, R.N.A. (2018). Increased Prefrontal Activity with Aging Reflects Nonspecific Neural Responses Rather than Compensation. The Journal of Neuroscience, 38, 73037313.

Murner-Lavanchy, I., Ritter, B. C., Spencer-Smith, M. M., Perrig, W. J., Schroth, G., Steinlin, M., Everts, R. (2014a). Visuospatial working memory in very preterm and term born children--impact of age and performance. Developmental Cognitive Neuroscience, 9, 106-116.

Murner-Lavanchy, I., Steinlin, M., Kiefer, C., Weisstanner, C., Ritter, B. C., Perrig, W., Everts, R. (2014b). Delayed development of neural language organization in very preterm born children. Developmental Neuropsychology, 39, 529-542. 
Narberhaus, A., Lawrence, E., Allin, M. P., Walshe, M., McGuire, P., Rifkin, L.,...Nosarti, C. (2009). Neural substrates of visual paired associates in young adults with a history of very preterm birth: Alterations in fronto-parieto-occipital networks and caudate nucleus. Neuroimage, 47, 18841893.

Perez-Roche, T., Altemir, I., Gimenez, G., Prieto, E., Gonzalez, I., Pena-Segura, J. L.,...Pueyo, V. (2016). Effect of prematurity and low birth weight in visual abilities and school performance. Research in Developmental Disabilities, 59, 451-457.

Salvan, P., Froudist Walsh, S., Allin, M. P., Walshe, M., Murray, R. M., Bhattacharyya, S.,...Nosarti, C.. (2013). Road work on memory lane-functional and structural alterations to the learning and memory circuit in adults born very preterm. Neuroimage, 15, Pt 1:152-161.

Smith, S.M., Nichols, T.E. (2009). Threshold-free cluster enhancement: addressing problems of smoothing, threshold dependence and localisation in cluster inference. Neuroimage, 44, 83-98.

Wilke, M., Holland, S.K., Altaye, M., Gaser, C. (2008). Template-O-Matic: a toolbox for creating customized pediatric templates. Neuroimage, 41, 903-913. 
Table 1. Characteristics of the group born very preterm and the control group.

Group born very preterm, $\mathrm{n}=37 \quad$ Control group, $\mathrm{n}=34$

Maternal education, $\leq 12 \mathrm{y} />12 \mathrm{y}, \mathrm{n}(\%)$

Paternal education, $\leq 12 \mathrm{y} />12 \mathrm{y}, \mathrm{n}(\%)$

Gestational age, weeks, mean (SD) min-max

Birth weight, g, mean (SD) min-max

Boys / girls, n (\%)

Brain pathology at term equivalent age

normal, n $(\%)$

minor, $\mathrm{n}(\%)$

major, n (\%)

Brain pathology at 12 years of age

normal, n (\%)

$32(86 \%)$

$5(14 \%)$

minor, $\mathrm{n}(\%)$

major, $\mathrm{n}(\%)$

0
29 (3) 24-33
$30(81 \%)$
$6(16 \%)$
$1(3 \%)$

$12(32 \%) / 23(62 \%)$

$23(62 \%) / 12(32 \%)$

$1160(345) 620-2120$

$23 / 14(62 \% / 38 \%)$

$$
\begin{aligned}
& 12(35 \%) / 16(47 \%) \\
& 12(35 \%) / 14(41 \%) \\
& 40(1) 37-42 \\
& 3624(472) 2830-4580 \\
& 16 / 18(47 \% / 53 \%)
\end{aligned}
$$

$34(100 \%)$

0

0 
Lind-Prematurity, Visual Perception and fMRI

Age at assessment, mean (SD) min-max

Handedness, right / left / ambivalent, n (\%)
12 y $9 \mathrm{~m}(6 \mathrm{~m}) 12$ y $2 \mathrm{~m}-13 \mathrm{y} 10 \mathrm{~m}$

$32 / 3 / 1(86 \% / 8 \% / 3 \%)$
$12 \mathrm{y} 7 \mathrm{~m}(3 \mathrm{~m}) 12 \mathrm{y} 2 \mathrm{~m}-13 \mathrm{y} 5 \mathrm{~m}$

33 / 1 / $0(97 \%$ / $3 \%$ / $0 \%)$

Data on handedness was missing for one prematurely born participant, data on maternal education for two prematurely born and six control participants, and data on paternal education for two prematurely born and eight control participants. 
Table 2. Accuracy (percentage and number of correct responses) and response times of correct responses in visual perception task, and differences between the group born very preterm and the control group.

\begin{tabular}{|c|c|c|c|c|c|}
\hline & $\begin{array}{l}\text { Group born very } \\
\text { preterm, } n=37\end{array}$ & $\begin{array}{l}\text { Control group } \\
\mathrm{n}=34\end{array}$ & $\mathrm{t}, \mathrm{df} / \mathrm{U}$ & P-value & $\begin{array}{l}\text { Cohen's d / } \\
\text { Eta squared }\end{array}$ \\
\hline Visual closure, correct responses, $\mathrm{n}$ & 36 & 34 & & & \\
\hline percentage, mean $(\mathrm{SD})$ & $71 \%, 12.7(2.2)$ & $70 \%, 12.7(3.3)$ & 549.5 & .457 & .008 \\
\hline median, $\min -\max$ & $13.0,7-16$ & $13.5,0-17$ & & & \\
\hline Visual closure, response time (seconds), $\mathrm{n}$ & 36 & 33 & & & \\
\hline mean $(\mathrm{SD})$ & $4.9(1.0)$ & $4.9(1.1)$ & 567.0 & .746 & .002 \\
\hline median, $\min -\max$ & $4.8,2.8-8.5$ & $4.9,3.0-7.5$ & & & \\
\hline \multicolumn{6}{|l|}{ Discrimination of a deviating figure, } \\
\hline correct responses, $\mathrm{n}$ & 37 & 34 & & & \\
\hline percentage, mean (SD) & $56 \%, 6.2(1.5)$ & $58 \%, 6.4(1.7)$ & 575.5 & .530 & .006 \\
\hline median, $\min -\max$ & $6.0,2-9$ & $6.5,0-9$ & & & \\
\hline \multicolumn{6}{|l|}{ Discrimination of a deviating figure, } \\
\hline response time (seconds), $\mathrm{n}$ & 37 & 33 & & & \\
\hline
\end{tabular}


Lind-Prematurity, Visual Perception and fMRI
mean $(\mathrm{SD})$
$4.8(0.9)$
$4.5(1.1)$
median, $\min -\max$
$4.6,3.0-7.0$
$4.3,2.7-7.2$

$-1.330,68$

.188

.300

Discrimination of figure and ground,

correct responses, $\mathrm{n}$

37

34

percentage, mean (SD)

$29 \%, 1.5(0.8)$

$28 \%, 1.4(0.8)$

610.5

.817

.001

median, $\min -\max$

$1.0,0-3$

$1.0,0-3$

Discrimination of figure and ground,

response time (seconds), $\mathrm{n}$

34

31

mean (SD)

$9.1(2.8)$

$8.8(2.8)$

513.0

.854

.001

median, $\min -\max$

$8.5,4.7-17.1$

$8.3,3.5-15.8$

Data on visual closure accuracy and response time was missing from one participant born prematurely. One control participant who did not give any correct responses in visual closure and

discrimination of a deviating figure tasks, and three control participants and three participants born prematurely who did not give any correct responses in discrimination of figure and ground,

were not included in analyses of response time of correct responses for corresponding series. 
Table 3. Regions with significantly stronger activation in the group born very preterm $(n=32)$ compared to the control group ( $\mathrm{n}=30$ ) during visual closure series. Locations and statistical values for cluster maxima are presented along with some sub-maxima to describe cluster extent.

Pediatric sample-specific brain template was used to spatially transform the functional magnetic resonance images.

\begin{tabular}{|c|c|c|c|c|c|c|}
\hline \multirow[t]{2}{*}{ Area } & \multirow[t]{2}{*}{ Cluster size } & \multirow[t]{2}{*}{ P-value (FWE) } & \multirow[t]{2}{*}{ TFCE value } & \multicolumn{3}{|c|}{ MNI coordinates } \\
\hline & & & & $\mathrm{x}$ & $\mathrm{y}$ & $\mathrm{z}$ \\
\hline Right thalamus & 228 & .008 & 523.73 & 6 & -3 & 15 \\
\hline Right thalamus & & .013 & 460.47 & 15 & -24 & 18 \\
\hline Left thalamus & & .024 & 410.12 & -12 & -15 & 18 \\
\hline Left caudate nucleus & & .018 & 432.18 & -9 & 3 & 15 \\
\hline Right caudate nucleus & & .039 & 350.06 & 15 & 9 & 6 \\
\hline Left temporal pole & 74 & .016 & 441.43 & -42 & 9 & -24 \\
\hline Left middle temporal & 24 & .039 & 350.25 & -63 & -6 & -12 \\
\hline Left pre- supplementary motor & 86 & .024 & 409.45 & -3 & 18 & 48 \\
\hline Left supplementary motor & & .041 & 346.19 & -12 & -3 & 54 \\
\hline Left dorsolateral prefrontal & 22 & .030 & 382.39 & -33 & 36 & 21 \\
\hline Left dorsolateral prefrontal & 17 & .039 & 348.91 & -24 & 33 & 48 \\
\hline
\end{tabular}


Lind-Prematurity, Visual Perception and fMRI

\begin{tabular}{|c|c|c|c|c|c|}
\hline Left dorsolateral prefrontal & 29 & .037 & 356.43 & -51 & 18 \\
\hline Left lateral motor/ premotor & & .042 & 344.90 & -48 & 9 \\
\hline Left dorsolateral prefrontal/ premotor & 6 & .048 & 334.57 & -27 & 15 \\
\hline Left orbitofrontal & 27 & .032 & 374.92 & -30 & 27 \\
\hline Left precuneus/ posterior cingulate & 14 & .032 & 375.13 & -15 & -51 \\
\hline Left anterior cingulate & 115 & .033 & 372.01 & -3 & 42 \\
\hline Left anterior cingulate & & .036 & 360.49 & -3 & 18 \\
\hline Left anterior cingulate & & .040 & 347.12 & -9 & 30 \\
\hline Left cerebellum & 46 & .035 & 362.75 & -18 & -84 \\
\hline Left cerebellum & & .037 & 354.81 & -33 & -75 \\
\hline
\end{tabular}

$\overline{\mathrm{FWE}}=$ family-wise error corrected for multiple comparisons, TFCE $=$ threshold-free cluster enhancement, $\mathrm{MNI}=$ Montreal Neurological Institute 
Infants born very preterm (birth weight $\leq 1500$ grams or gestational age $<32$ weeks) between January 2001 and December 2006 in the Turku University Central Hospital, $\mathrm{n}=290$

Infants who lived in the hospital catchment area and whose parents spoke and understood written Finnish or Swedish, $n=276$

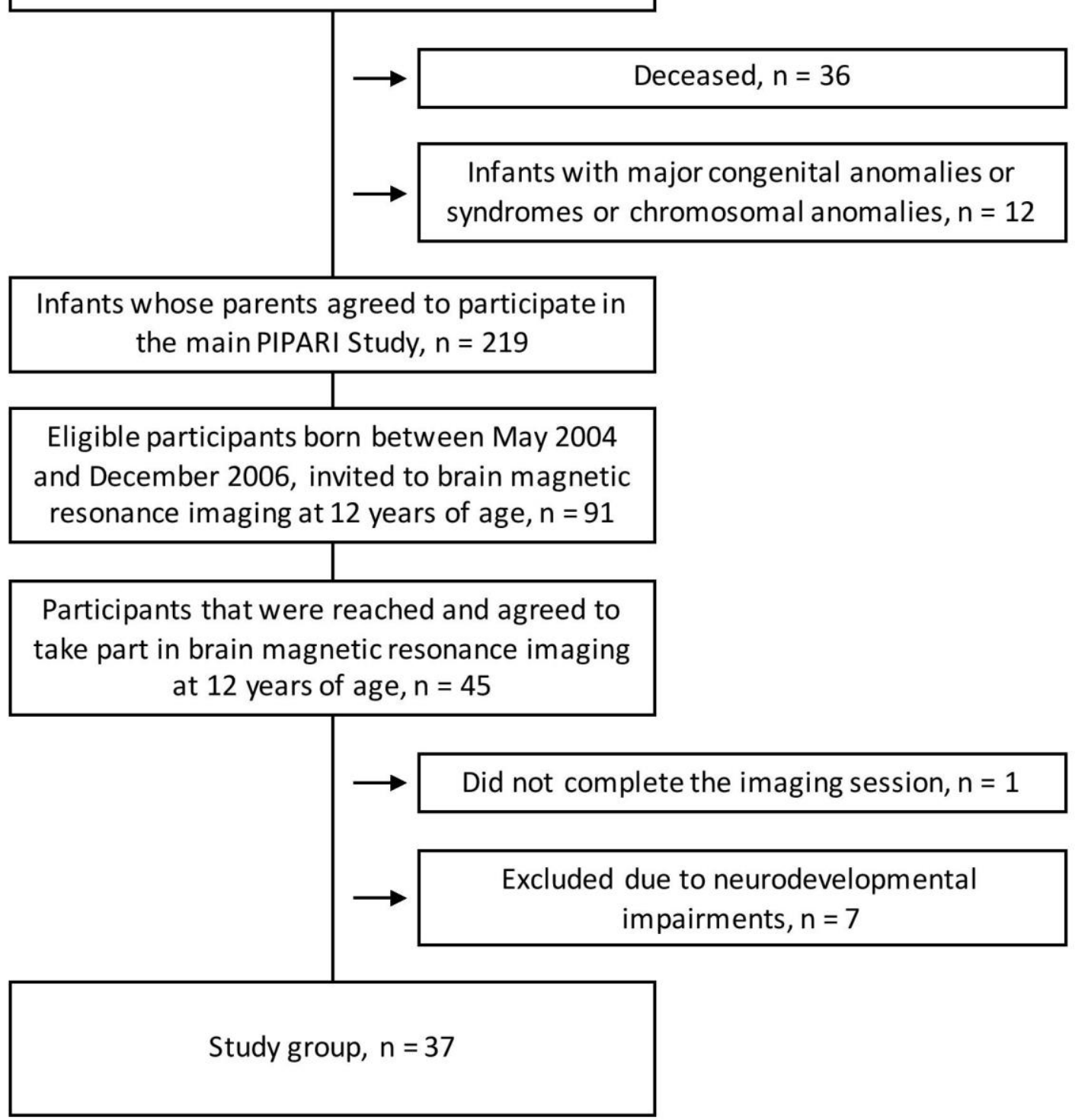

Figure 1. A flow chart of the study population born very preterm. 


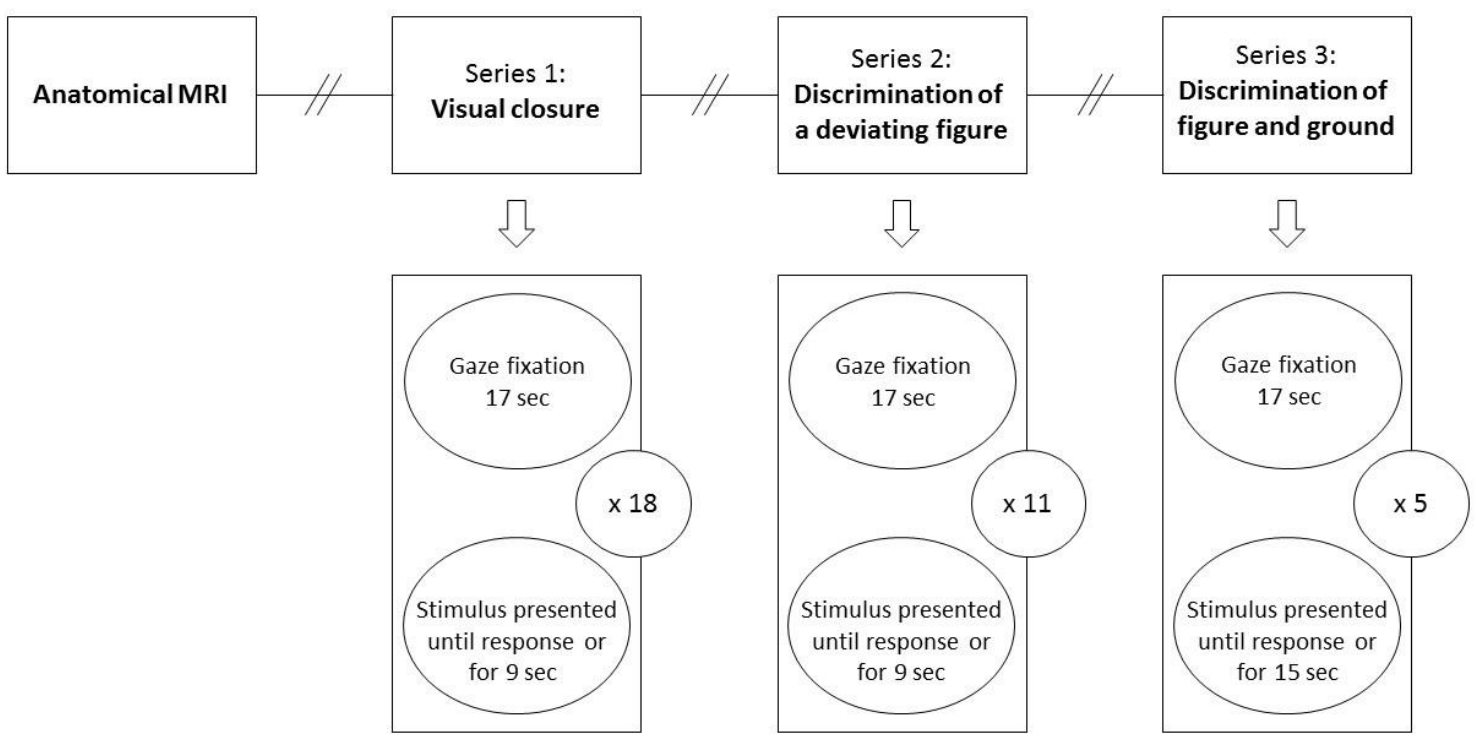

Figure 2. A flow chart of the visual tasks. Each task series was divided into a different number of items. The stimuli alternated with gaze fixation. The duration of fixation was constant, but the item ended when the child answered or when the time limit had passed. The instructions given to the child are marked with "//" and were presented according to the manual. 


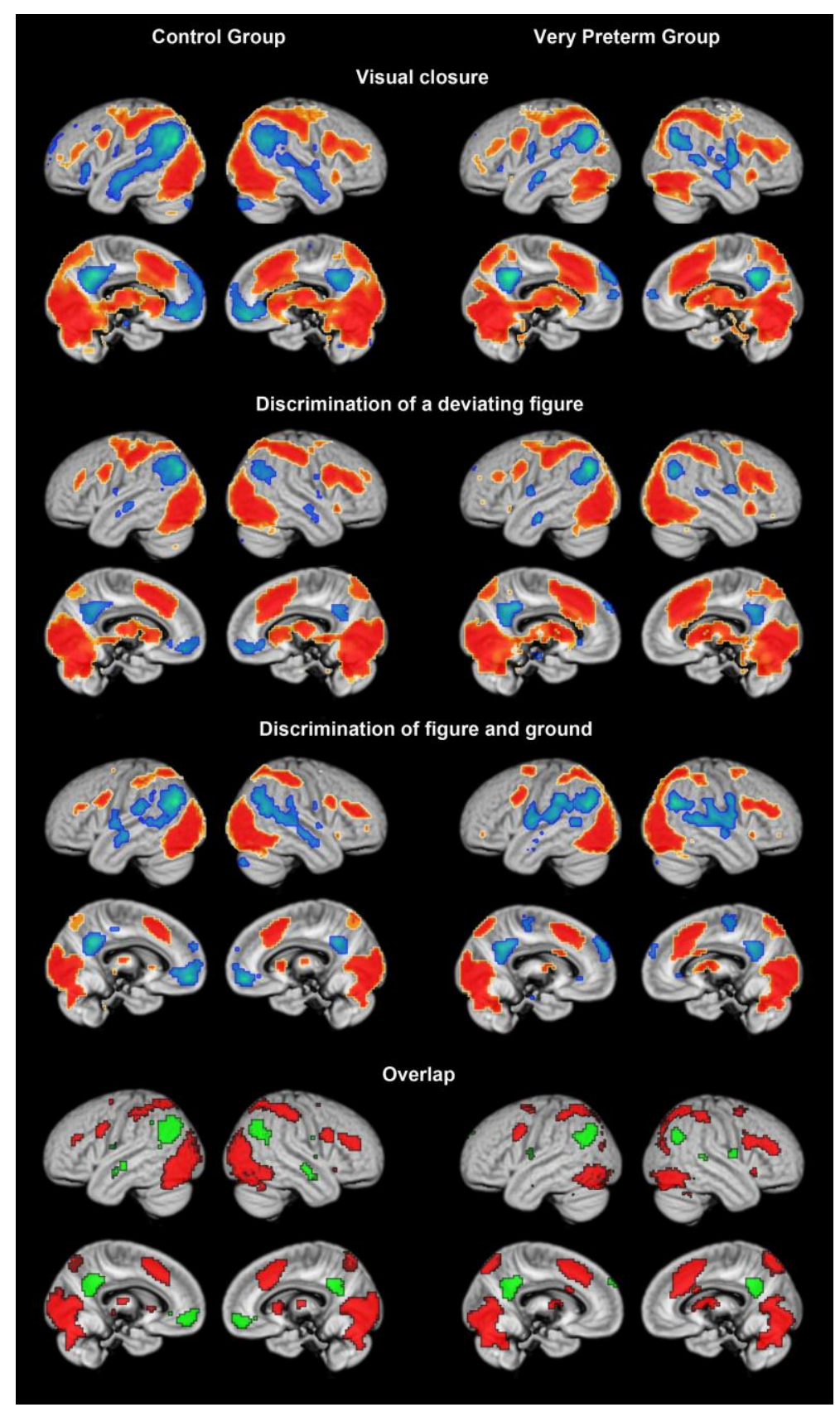

Figure 3. Significant ( $p<0.05$ corrected voxel threshold) activation (hot colors) and deactivation (cold colors) patterns in the control group and the group born very preterm, across the three visual perception tasks obtained by one sample t-tests comparing the task and gaze fixation conditions. The bottom row shows overlapping areas across all tasks within each group obtained by inclusive masking. The activation differences are shown on the rendered surface of the customized gray matter template and projection depth of $12 \mathrm{~mm}$ from the surface. 


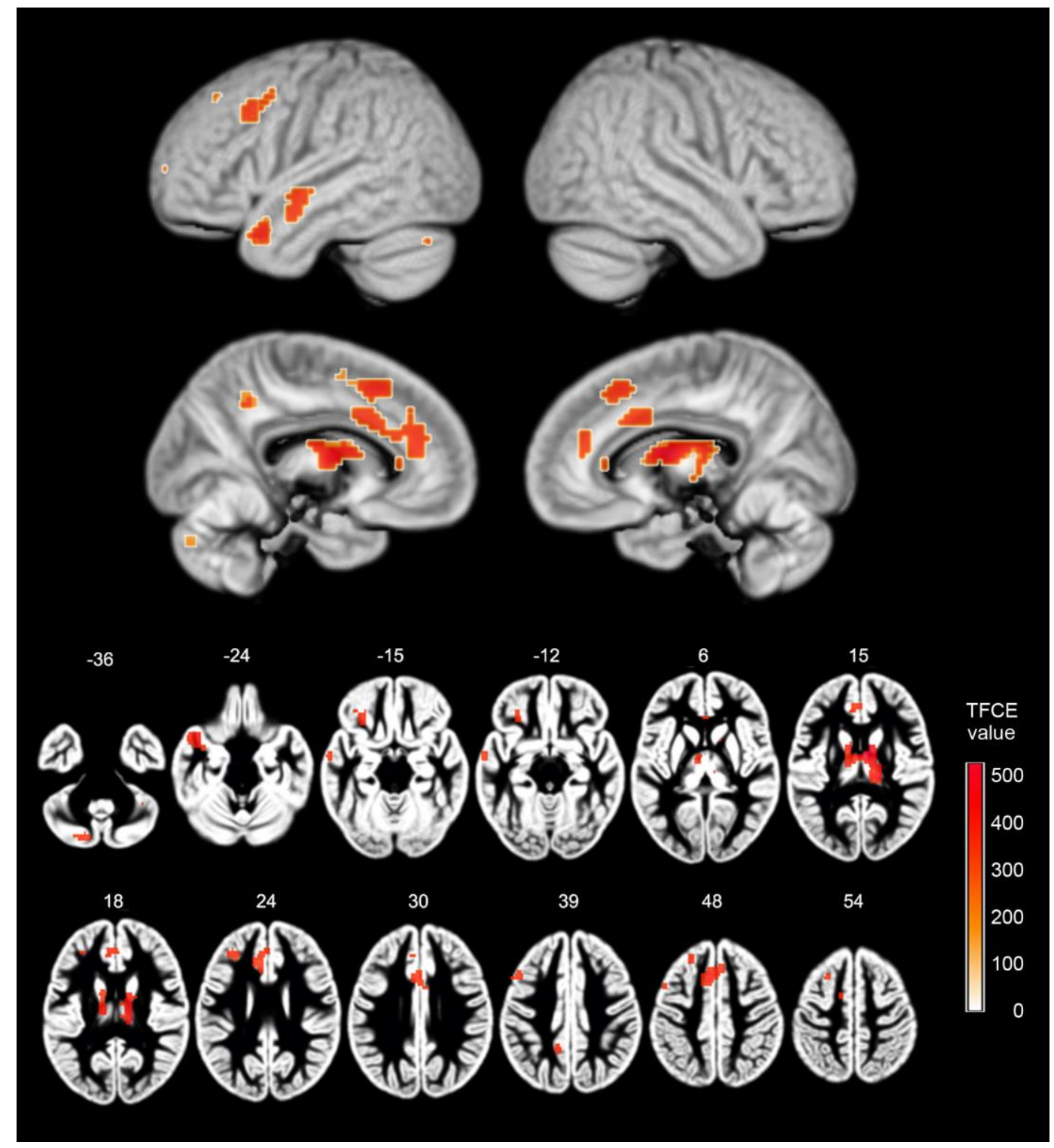

Figure 4. Areas of significant $(\mathrm{p}<0.05$, family-wise error corrected, threshold-free cluster enhancement statistic) group difference (prematurely born > controls) obtained in the visual closure task. The results are shown overlaid onto brain surfaces with $12 \mathrm{~mm}$ search depth (top of the figure) and a series of axial slices (bottom of the figure) of the sample-specific grey matter template. Images are oriented according to the neurological convention. The position of each slice is indicated by a number (z Montreal Neurological Institute coordinate). 